\title{
ROBOTIC REHABILITATION DEVICES OF HUMAN EXTREMITIES: DESIGN CONCEPTS AND FUNCTIONAL PARTICULARITIES
}

\author{
Suren Sargsyan \\ IRCCyN, 1 rue de la Noë, BP \\ 92101, 44321 Nantes Cedex 03, \\ France \\ Suren.Sargsyan@irccyn.ec-nantes.fr
}

\author{
Vigen Arakelian \\ Department of M\&CSE, \\ I.N.S.A. Rennes \\ 20 av. des Buttes de \\ Coesmes, CS 70839, 35708 \\ Rennes, France \\ vigen.arakelyan@insa-rennes.fr
}

\author{
Sébastien Briot \\ IRCCyN, 1 rue de la Noë, BP \\ 92101, 44321 Nantes Cedex 03, \\ France \\ Sebastien.Briot@irccyn.ec-nantes.fr
}

\section{ABSTRACT}

All over the world, several dozen million people suffer from the effects of post-polio, multiple sclerosis, spinal cord injury, cerebral palsy, etc. and could benefit from the advances in robotic devices for rehabilitation. Thus, for modern society, an important and vital problem of designing systems for rehabilitation of human physical working ability appears. The temporary or permanent loss of human motor functions can be compensated by means of various rehabilitation devices. They can be simple mechanical systems for orthoses, which duplicate the functions of human extremities supplying with rigidity and bearing capacity or more complex mechatronic rehabilitation devices with higher level of control. We attempt to cover all of the major developments in these areas, focusing particularly on the development of the different concepts and their functional characteristics. The robotic devices with several structures are classified, taking into account the actuation systems, the neuromuscular stimulations, and the structural schemes. It is showed that the problems concerning the design of rehabilitation devices are complex and involve many questions in the sphere of biomedicine, mechanics, robot technology, electromechanics and optimal control. This paper provides a design overview of hardware, actuation, sensory, and control systems for most of the devices that have been described in the literature, and it ends with a discussion of the major advances that have been made and should be yet overcome.

\section{INTRODUCTION}

Applications of robotics systems include several fields such as industrial production, inspection and quality control, field service, surveillance, as well as medicine and health care. The last area is particularly important due to the ability of medical robots to significantly improve surgeons' technical capability, either by making existing procedures more accurate, faster, or less invasive. Similarly, robotic systems for rehabilitation or for assistance in daily living offer real advantages. In the last decade, the interest in this field has raised mainly due the growing demand caused by increasing number of stroke patients.

In this paper, an overview of rehabilitation devices of human extremities taking into accounts their design concept and functional particularities are presented. The described rehabilitation devices of human extremities are presented by two principal groups: stationary and mobile. It has been considered that the rehabilitation system is stationary if the orthosis, i.e. the device intended to correct or improve the locomotive function, is mounted on the immobile support and mobile if the orthosis is mounted on the movable support. Let us start the overview from the analysis of stationary rehabilitation devices.

\section{STATIONARY REHABILITATION DEVICES OF HUMAN EXTREMITIES}

The stationary rehabilitation devices can be distinguished by several criteria. One of the most important distinctive characteristics of rehabilitation devices is the nature of the compensation force, which can be passive or active. The compensation force can be considered as passive if it is created by a non-motorized element.

Let us consider the working principle of such a rehabilitation device called the Ergys2 system. The Ergys2 (Therapeutic Alliances Inc, Ohio, USA) is a rehabilitation system for treating neuromuscular disorders and diseases (Fig. 1). It uses computerized functional electrical stimulation (CFES) to allow people with little or no voluntary leg movement to actively pedal [1].

Computer generated, low-level electrical pulses transmitted through surface electrodes cause coordinated contractions of 
the leg muscles: quadriceps, hamstring, and gluteus. Sensors located in the Ergys 2 provide continuous feedback to a computer which controls the sequence of muscle contractions as well as resistance to pedaling. The result is smooth and natural pedaling with the leg muscles supplying the power.

The Ergys2 provides significant benefits for both paraplegics and quadriplegics. It prevents muscle atrophy, relaxes muscle spasms, improves circulation and increases range of motion [2].

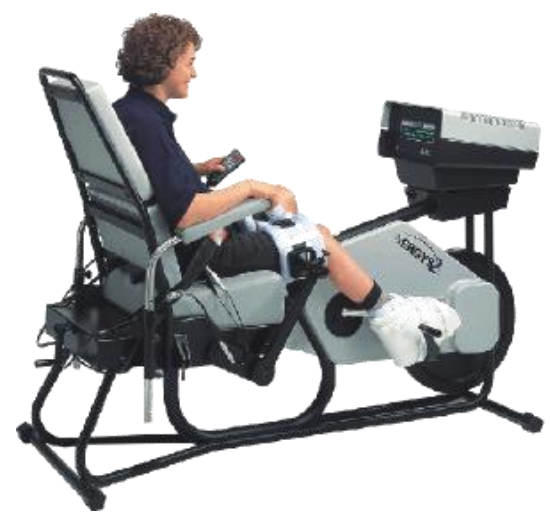

Figure 1. Ergys rehabilitation system.

However, the working principle of most stationary rehabilitation devices is based on the motorized orthosis, which can be with electrosimulation or without it. Let us consider some examples.

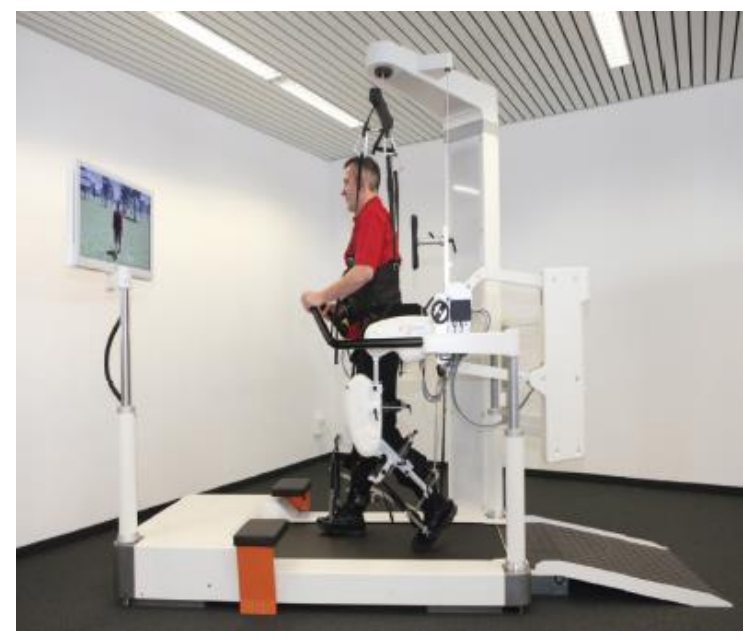

Figure 2. Lokomat system.

The Lokomat (Hocoma AG, Volketswil, Switzerland) consists of a robotic gait orthosis and an advanced body weight support system (Fig. 2) [3]. This system adds the ability to measure the patient's activity by a way of force transducers fitted directly on the motors and offers the possibility to adjust the level of gait assistance for each leg between full and zero guidance force.
The LokoHelp (LokoHelp Group) is an electromechanical device developed for improving gait after brain injury [4]. The LokoHelp (Fig. 3) is placed in the middle of the treadmill surface parallel to the walking direction and fixed to the front of the treadmill with a simple clamp. It also provides a body weight support system for the patient. Clinical trials have been conducted to analyze its feasibility and efficacy [5][6]. The results show that the system improves the gait ability of the patient in the same way as the manual locomotor training. However, using the LokoHelp less therapeutic assistance is required.

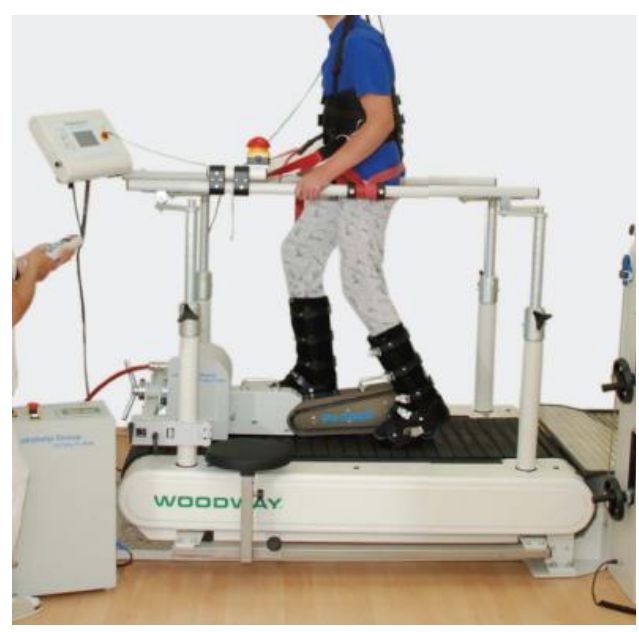

Figure 3. LokoHelp gait trainer PEDAGO.

The aim of MotionMaker (Cyberthosis, Switzerland) (Fig. 4) is to maintain, or increase muscular volume and capacity for patients in the chronic phase, to reinstate mobility of the joints, to train cardiac capacity and prevent neurological osteoporosis. This innovative concept not only allows automation of the treatment, but also an active participation of the patient's muscles.

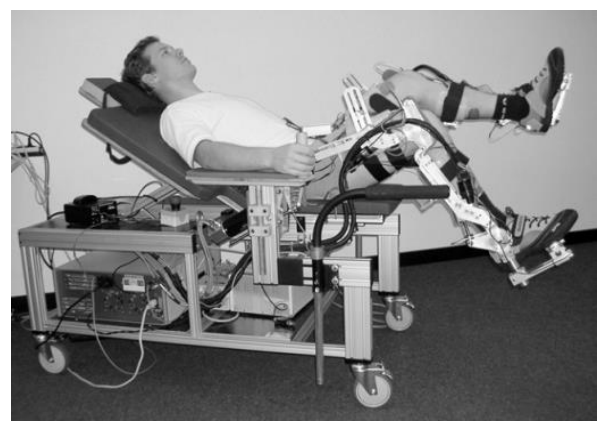

Figure 4. MotionMaker rehabilitation device.

The patient's limbs can be mobilized passively or work actively in an electro-stimulated manner and/or voluntarily against resistance loads created by the motors [7].

The MotionMaker has shown the advantages of combining robotics and controlled electrostimulation to improve voluntary 
control [8]. The activation of muscles in exercises of neurological re-education brings patients not only the recovery of muscle strength and joint mobility, but also the proprioceptive information essential for recovering the ability to activate by themselves their paralysed muscles.

Two other robotic systems have been developed with a similar working principle: (i) the Lambda [9], a rehabilitation and fitness robot used for mobilization of lower extremities that provides the movement of the lower extremities in the sagittal plane, including an additional rotation for the ankle mobilization; and (ii) a wire-driven leg rehabilitation system [10] developed by the National Institute of Advanced Industrial Science and Technology (AIST) of Tsukuba.

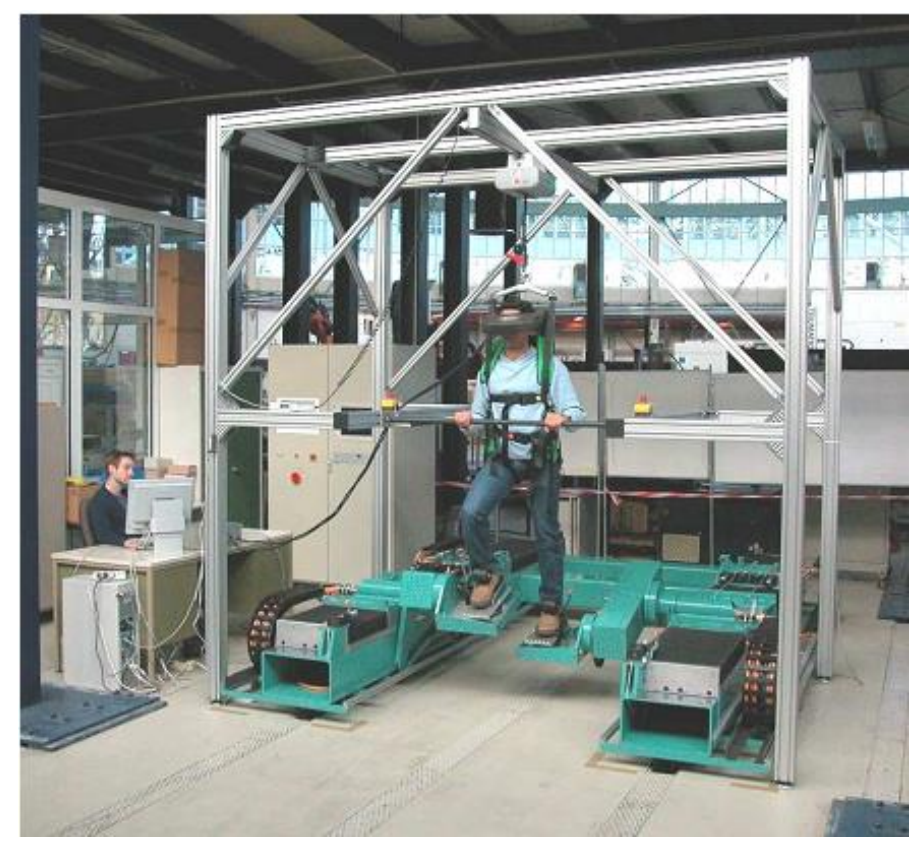

Figure 5. HapticWalker robotic walking simulator.

The HapticWalker [11] comprises two programmable foot platforms with permanent foot machine contact (Fig. 5). One of the applications of the machine is the gait rehabilitation for neurologically impaired persons such as afterstroke or spinal cord injury.

The machine is equipped with electrical direct drive motors, enabling highly dynamic footplate motions. For force feedback 6-degrees-of-freedom force/torque sensors are mounted under each foot platform.

The HapticWalker is designed as a scalable and modular system with a unit-by-unit extensibility. The basic unit enables movements in 3-degrees-of-freedom per foot in the sagittal plane. Each footplate can be extended to up to 6-degrees-offreedom, plus an additional metatarsal joint drive. The system is able to simulate not only 'smooth' trajectories like walking on plane floor, stepping staircases up/down, but also walking on rough ground or even stumbling. The machine is controlled by a full-featured robot control software and hardware.
The Lopes [12] combines a freely translatable and 2-Dactuated pelvis segment with a leg exoskeleton containing three actuated rotational joints: two at the hip and one at the knee (Fig. 6). The joints are impedance controlled to allow bidirectional mechanical interaction between the robot and the training subject. Evaluation measurements show that the device allows both a "patient-in-charge" and "robot-in-charge" mode, in which the robot is controlled either to follow or to guide a patient, respectively. Electromyography (EMG) measurements (one subject) on eight important leg muscles show that free walking in the device strongly resembles free treadmill walking; an indication that the device can offer task-specific gait training.
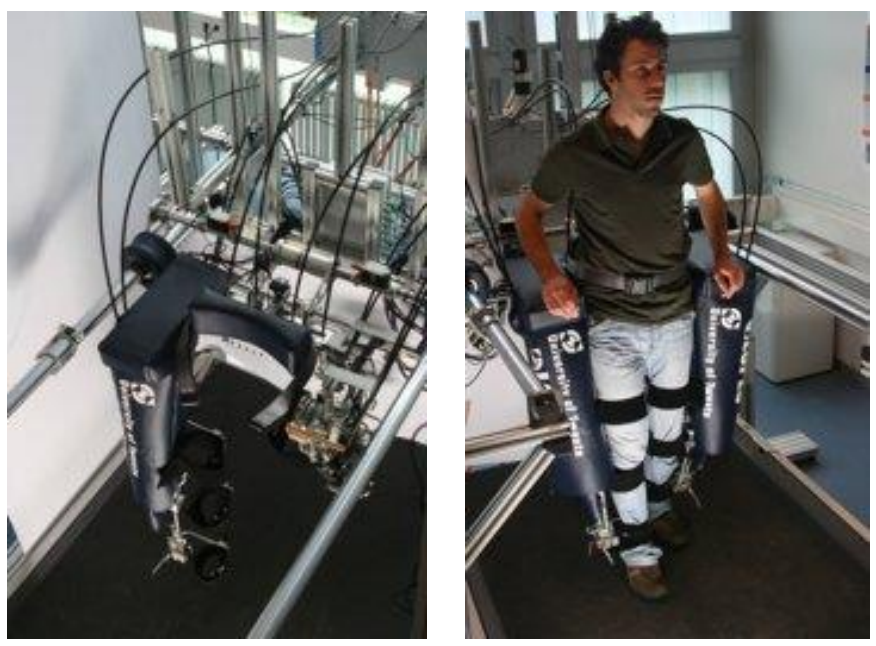

Figure 6. Lopes gait rehabilitation device.

The Gait Trainer GT I (Reha-Stim, Berlin, Germany) [13] has been created to improve the patient's ability to walk through continuous practice. The Gait Trainer GT I assists the patient in his or her recovery of gait by relieving the body of its own weight and adapting the pace to the individual ability of the patient. The patient is being positioned using the built-in swivel device, the feet getting fixed on the base plates and the wire mounts attached in order to compensate the body's side movement. The Gait Trainer GT I is attached to the bottom of the foot and does not provide the abrupt transition in ground reaction force that normally occurs between swing and stance. Step length and gait speed are adjustable. During therapy, the integrated servo drive automation supports the patient's own effort in order to keep the rotation speed constant. Horizontal and vertical trunk movements of the patient are also assisted according to the gait phase. The Gait Trainer GT I allows flexible deployment of its treatment for a number of diseases such as CP-children, patients with incomplete paraplegia, SHT, MS and Parkinson, also patients with joint replacements.

The AutoAmbulator (HealthSouth Cooperation) [14] is a body-weight supported treadmill robot system. Robotic arms are strapped to the patient's legs at the thigh and ankle, driving them through a stepping pattern. While using the AutoAmbulator, patients can walk because the device holds 
their body upright while mechanized braces help guide their legs across the treadmill. A touch screen computer monitors knee torque, thigh torque, and speed, among other session data. An automatic shut down feature is triggered through severe spasms or an improper foot strike on the treadmill by the patient. It may help all sorts of patients with central nervous system disorders. AutoAmbulator is equipped with safety interlocks, redundant travel limits, variable torque limits, and a safety light array to detect improper foot drop. The AutoAmbulator is an exciting technological advance in physical therapy, but it is not a cure for walking impairments [15].

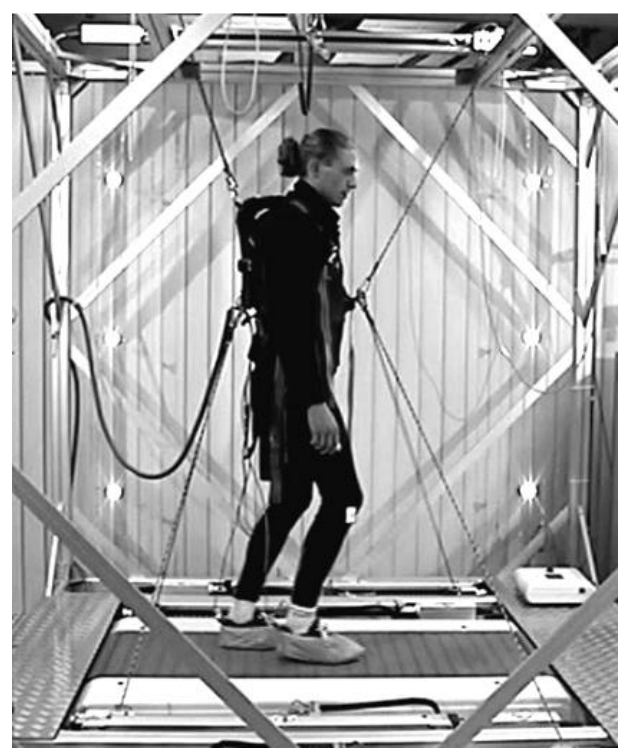

Figure 7. String-Man rehabilitation robot.

The ARTHUR (Ambulation-assisting Robotic Tool for Human Rehabilitation) [16] is a 2-degrees-of-freedom robot that makes use of linear electric direct-drive motors to precisely apply forces to the leg during stepping. It was designed primarily to conduct research on human motor control of gait and has been successfully used with unimpaired participants to create novel dynamic environments that can enhance motor adaptation[16]. ARTHUR cannot assist at both the knee and the lower shank as experienced therapists commonly do.

The PAM (Pelvic Assist Manipulator) [17] is a 5- degreesof-freedom compliant robot that accommodates naturalistic motion of pelvis during walking actuated by six pneumatic cylinders, which, combined with a nonlinear force-tracking controller, provide back drivability and large force output at a relatively low cost. PAM can act as a teach-and-replay device with a PD position controller driving the pelvis onto the reference trajectory specified with or without the help of therapists.

The POGO (Pneumatically Operated Gait Orthosis) is a leg robot aimed at the goal of being used in the clinic for daily locomotor [18]. POGO is composed of two pneumatic cylinders that are fixed to a telescoping rail system that extends from a hip belt to a molded cuff around the lower shank. One cylinder pushes and pulls between the hip belt and the rail, thus flexing and extending the hip. A second cylinder pushes and pulls between the middle of the rail and a strap wrapped just below the knee, thus providing knee flexion and extension.

The String-Man is a wire robot for manipulating the torso of the patients based on the string-puppet principle [19]. Seven wires connected to the patient's trunk achieve weight bearing and 6-dof manipulation (Fig. 7). Force sensors between the wires and the actuators allow force or impedance control: this permits adjustment of the interaction control from totally passive to completely active and allows the patients to test their balancing capabilities. The String-Man can control both the zero-moment-point location and the ground reaction force, using foot force sensors in addition to the wire force sensors.

\section{MOBILE REHABILITATION DEVICES OF HUMAN EXTREMITIES}

The most important feature of the mobile rehabilitation devices is the fact that it is easily portable and wearable. The compact and lightweight characteristics of these advanced rehabilitation devices allow them to be used in an average chair, while standing, or perhaps even during ambulatory motion.

Passive gravity balancing orthoses have been proposed for the upper arm [20]-[23]. In the studies [24], [25], a design of an exoskeleton for full or partial gravity-balancing of a human leg during motion has been proposed (Fig. 8).
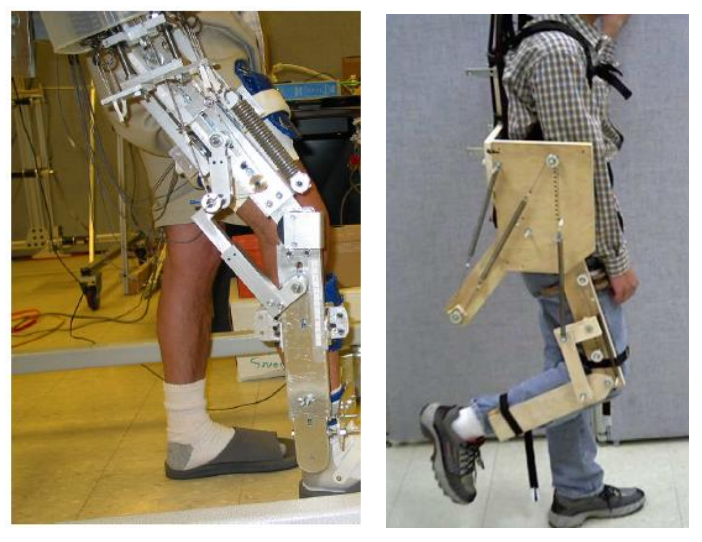

Figure 8. Prototypes developed in the University of Delaware.

The device is worn by the user and segments of the exoskeleton are strapped to the corresponding segments of the human leg.

In order to improve the compensation in the orthoses several balancing approaches have been developed [26]-[29]. In these studies, the spring mass has been included in the balancing conditions that improves the quality of balance [30], [31].

Recently, the orthosis ALEX along with force-field controller has been developed for gait rehabilitation of stroke survivors. Its aim is to help in retraining the gait pattern by using assist-as-needed approach which allows the patient to 
participate more actively in the retraining process compared to other currently available robotic training devices (Fig. 9) [32].

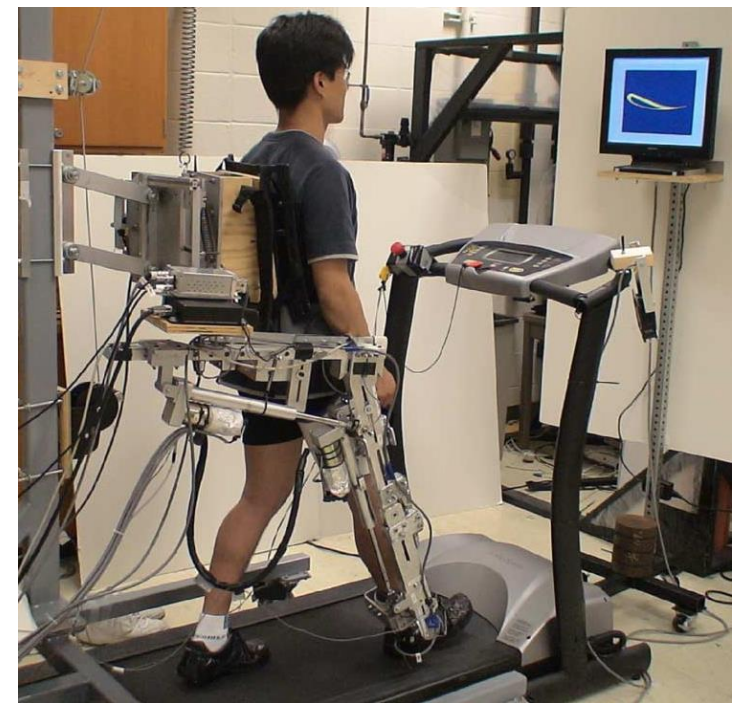

FIGURE 9. Motorized orthosis ALEX.

KAFO (knee-ankle-foot orthoses) (Fig. 10) and HKAFO (hip-knee-ankle-foot orthoses) have been used for the treatment of patients with paraplegia after thoracic and/or lumbar spinal cord injuries [33].

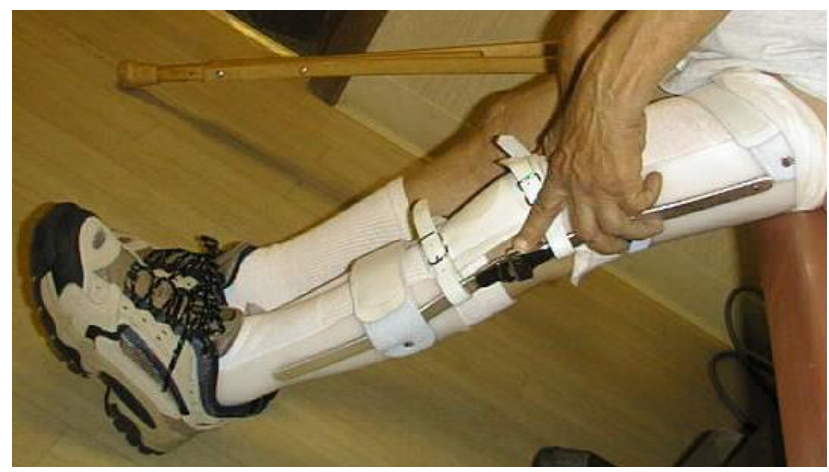

Figure 10. Kafo.

While these orthoses provide excellent stability during steady-state standing, they are not convenient for daily home use. As a result, the rate of continuous use of orthoses is low, and they tend to be worn only for standing and exercise in the home setting. Even in the clinical setting, these orthoses are not frequently used because of problems associated with their weight, size, appearance, and comfortableness [34].

The Mobility Device (Tempe, Arizona, USA) allows patients with a wide range of impairments to walk over ground safely (Fig. 11). It is enforced with a battery operated power lift actuator that lifts patients: weighing up to $159 \mathrm{~kg}$ (350lbs), from sit to stand. To make the effort easier, the Mobility Device is designed to assist both patient and therapist [35].

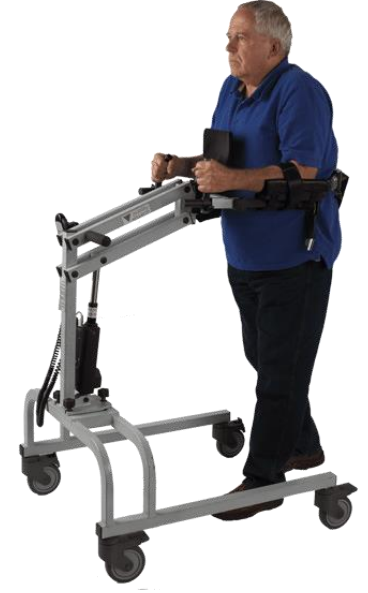

Figure 11. Mobility Device.

The Walking Trainer (Ropox A/S, Naestved, Denmark) is a device to provide a normal walking pattern by controlling weight bearing, balance and posture during walking therapy (Fig. 12). It can lift patient from sitting position, with the help of attached to the device lifting actuator. Training can normally be done by one therapist [36].

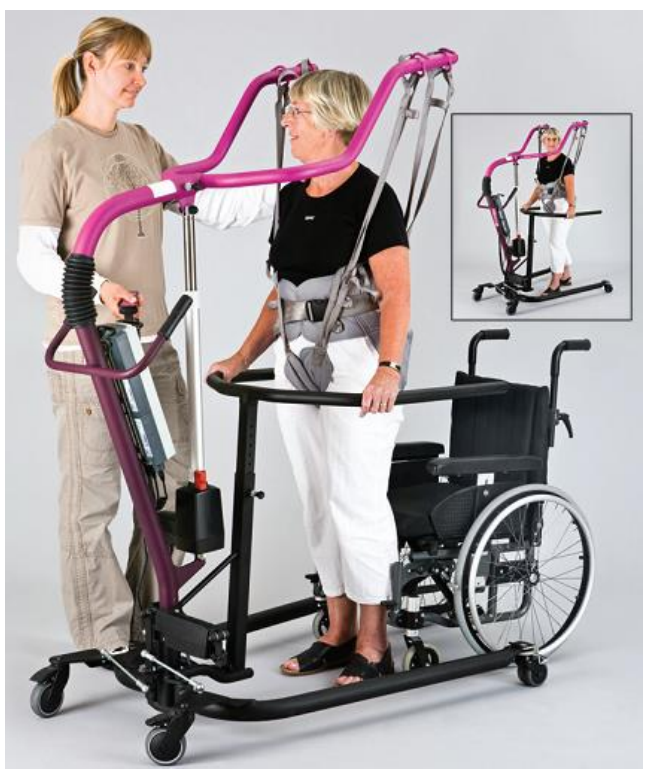

Figure 12. Walking Trainer.

The Erigo (Hocoma AG, Volketswil, Switzerland) is an innovative tilt table with integrated robotic stepping functions (Fig. 13). It combines simultaneous dynamic leg movement and physiological loading of the lower extremities of long bed rest patients or of neurological patients in the early phase of rehabilitation. The purpose of the Erigo is to provide the opportunity of intensive movement therapy and physiological loading of the lower limbs at an early stage after onset 
combined with the possibility of simultaneous up-righting of the patient [3].

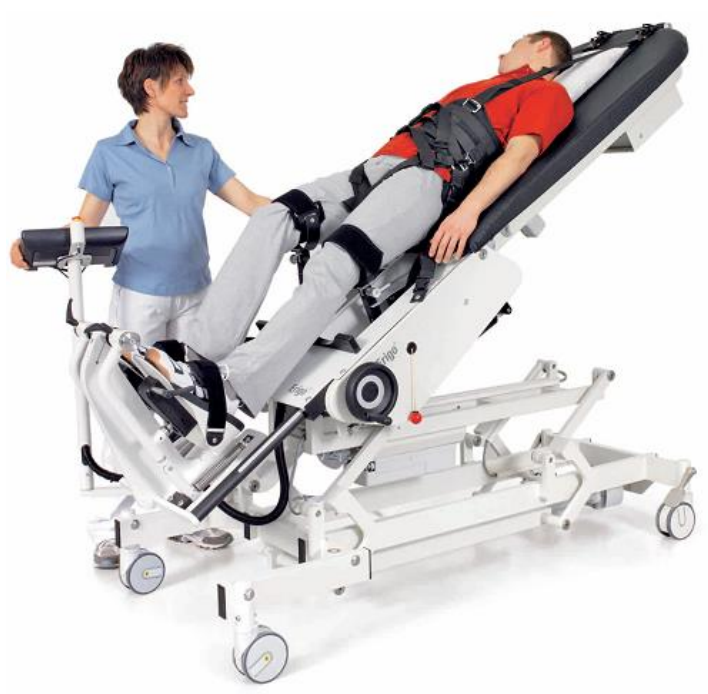

Figure 13. Erigo.

The WalkTrainer (Cyberthosis, Monthey, Lausanne, Switzerland) is equipped with orthoses for the legs and pelvis, body weight support, and with CLEMS (Closed Loop Electrical Muscle Stimulation) [7], this system allows near perfect mimicking of walking movements with the aim of stimulating nervous system plasticity (Fig. 14). The WalkTrainer is composed of body weight support, active winch, harness, pelvic orthesis, gait orthesis, motorized wheels, battery, electrostimulator, electrodes and control unit. The main function of the walking frame is to follow the patient while moving. It serves as a mobile support for all the other components. Training with WalkTrainer should allow one to go even further in the relearning of gait patterns by offering overground walking with controlled pelvic motion and muscle activity. The main difficulty for this system is to propose an alternative at least as good as the wheelchair in functional terms, but also in terms of ease of use and implementation.

The LiteGait (Tempe, Arizona, USA) [35] is a gait training device that simultaneously controls weight bearing, posture, and balance over a treadmill or over ground. It consists of a yoke, straps, a harness, an actuator and a base. The lift mechanism is a rechargeable, battery powered actuator which permits the incremental adjustment of weight bearing. This allows patient to be brought to an upright posture.

The handheld control allows therapists to conveniently bring patient to a standing position and adjust the amount of weight supported. LiteGait provides proper posture, reduces weight bearing, eliminates concerns for balance, and facilitates the training of coordinated lower extremity movement. It permits unilateral or bilateral support, allowing progression of the weight bearing load from non to full weight bearing and also allows the clinician to manually assist the legs and pelvis to achieve proper gait patterns. LiteGait also provides posture and balance control, creating a secure, fall free environment for the patient.

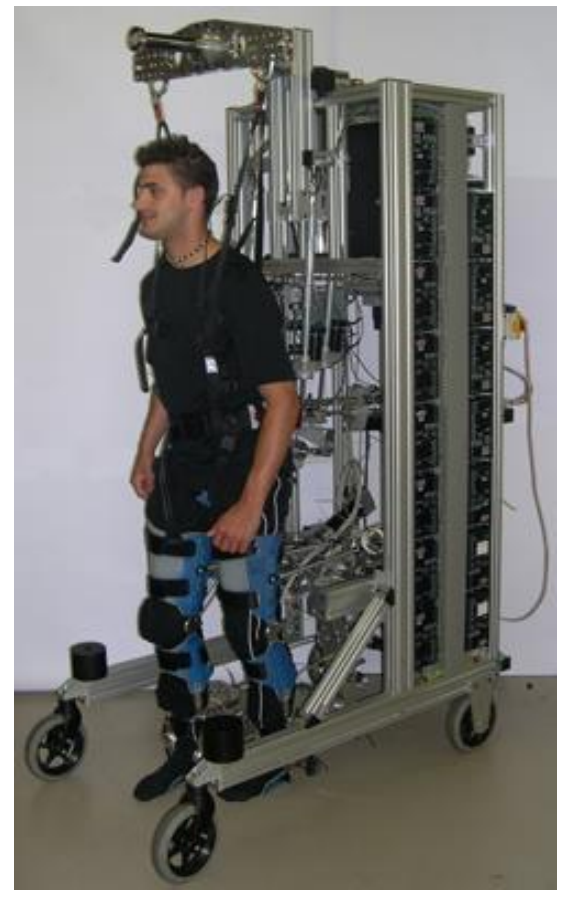

Figure 14. WalkTrainer.

The Parastep (Sigmedics, Inc, Fairborn, Ohio, USA) is a microcomputer controlled functional neuromuscular stimulation (FNS) system that enables independent, unbraced ambulation by people with a spinal cord injury. The computer is programmed to control the sequence of muscle contractions in the lower extremities that enable the functions of sit-to-stand, right and left step and stand to sit. Users are taught to initiate functions by activating commands through switch modules mounted on either side of the walker handle bars [37].

The BerkelBike (BerkelBike BV, Sint-Michielsgestel, Netherlands) [38] is a combination of a hand and a recumbent bike and is driven by arm as well as leg power (Fig. 15). It provides support to both muscle groups helping to rehabilitate and keep the muscles stronger and fitter. The benefits of cycling for impaired patients are to overcome overweighting due to lack of activities. The BerkelBike is for patients with spinal cord injury, polio, multiple sclerosis or stroke (CVA). The BerkelBike (Classic, Home or Pro) is equipped with a sensor that tells the Impuls stimulator the position of the legs. The software in the stimulator uses this information to calculate which muscles to stimulate and the strength of electric pulse required. The electric pulses generated are passed to the muscles via a pair of Impuls electrode shorts. These are like cycling shorts with a number of electrodes embedded within them. The electric pulses stimulate the muscles, causing them to contract at the appropriate time, allowing the person to cycle again [38]. 


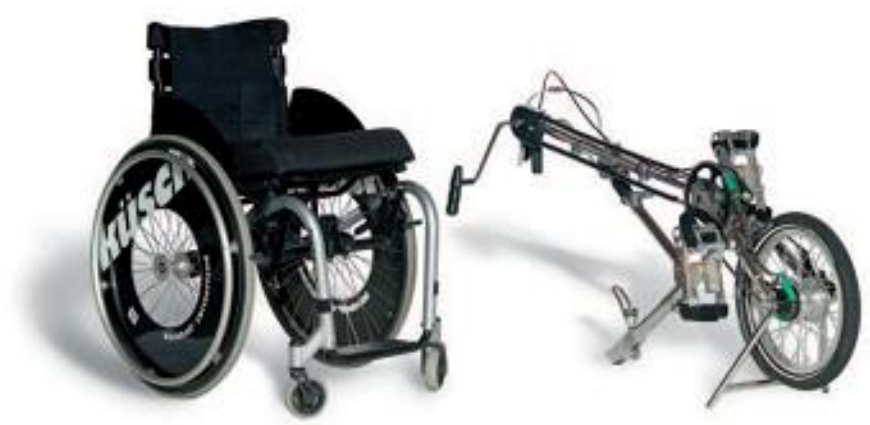

Figure 15. Berkelbike classic.

The Walkabout (Polymedic Co., Goldcoast, Queensland, Australia) [33] functions as a single-axis hip joint connecting two KAFOs (Fig. 16). The unit is positioned and attached to the KAFOs between the thighs, under the perineum, but is also removable. The Walkabout system provides stability during standing and walking, is compatible with wheelchairs, have cosmetic benefits, and is easy to don/doff. However, the Walkabout system has a hypothetical axis that is lower than the position of the physiological hip joint. It was suggested that this discrepancy would create a significant impediment to ambulation by increasing resistance to the movement of the legs.
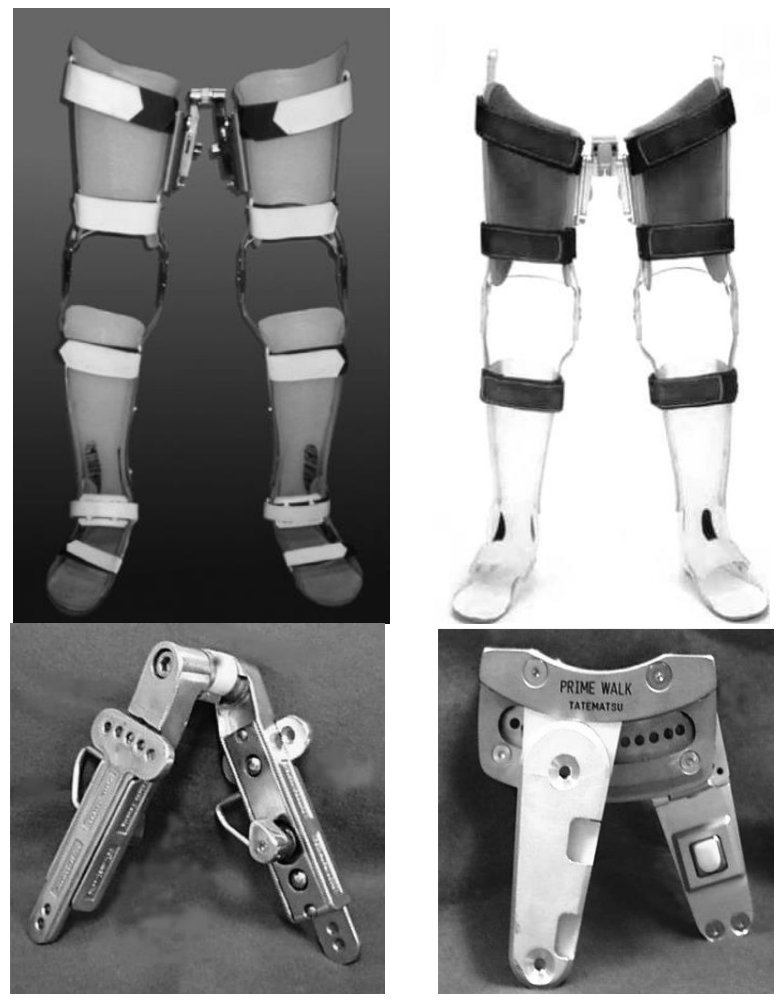

Figure 16. Walkabout, Primewalk.
The Primewalk (TIMS Co., Fukamiiwahana, Fujiko-cho, Nishikamo, Aichi, Japan) [33] is a KAFO with another medial linkage device, which has a virtual axis based on a sliding mechanism (Fig. 16). The Primewalk device is a sliding-type medial hip joint that had 12 embedded bearings in order to ensure smooth movement[33]. It is possible to wear the Primewalk system on a wheelchair. The average gait velocity is higher, cadence is faster, and stride is longer with the Primewalk system than with the Walkabout system. The value for the step length divided by the pelvic rotation angle is larger with the Primewalk system than with the Walkabout system. The Primewalk system improved the gait efficiency by the sliding mechanism that set the virtual axis of the orthoses hip joint higher than that of the hinge-type joint.

The RoboKnee (Yobotics, Inc., Cincinnati, OH, USA) is a 1-degree-of-freedom powered knee exoskeleton, for adding power at the knee to assist in stair climbing and squatting during load-carrying (Fig. 17) [39]. The RoboKnee may be used for the patients who are suffering from weakness in their lower extremities, such causes including cerebral vascular trauma (stroke), post-polio syndrome, multiple sclerosis, muscular dystrophy, and aging. The device consists of a linear series-elastic actuator connected to the upper and lower portions of a knee brace, with the help of which low impendence is achieved just below the hip and on the calf, respectively [40].

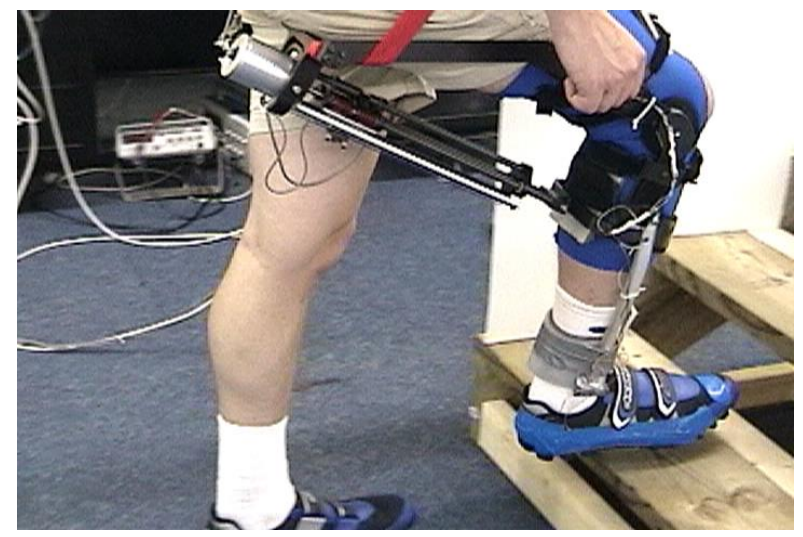

Figure 17. RoboKnee.

The control of the RoboKnee utilizes the ground reaction force and the center of pressure in the sagittal plane to create a positive feedback force amplification control scheme of the torque at the knee.

The Knee Exoskeleton [41] can be applied to the patients who suffer some form of leg weakness (Fig. 18). The concept of powered orthotic devices is to harness the passive dynamics of locomotion to reduce energetic requirements. An energetically-autonomous powered knee exoskeleton is designed to facilitate running. The device consists of a knee brace in which a motorized mechanism actively places and removes a spring in parallel with the knee joint. This mechanism is controlled such that the spring is in parallel with 
the knee joint from approximately heel-strike to toe-off, and is removed from this state during the swing phase of running. In this way, the spring is intended to store energy at heel-strike which is then released when the heel leaves the ground, reducing the effort required by the quadriceps to exert this energy, thereby reducing the metabolic cost of running.

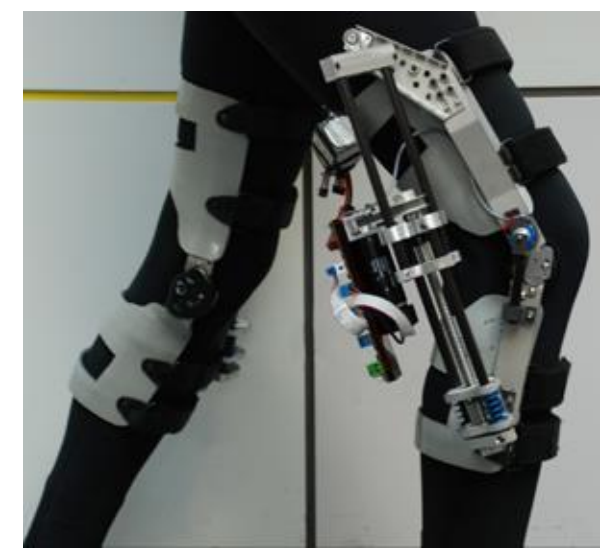

Figure 18. Knee exoskeleton.

Hybrid Assistive Limb (HAL) (CYBERDYNE Inc, Japan) [42] consists of controller, computer, battery, bioelectrical sensors, angular sensors, acceleration sensors, floor reaction force sensors. It requires $\mathrm{AC} 100 \mathrm{~V}$ electricity and can operate continuously approximately 2 hours 40 minutes. The total weight of the device is $21 \mathrm{~kg}$. It supports activities like standing up from a chair, walking, climbing up and down stairs, holding and lifting heavy objects up to $70 \mathrm{~kg}$. HAL uses biosensors from the bio signals to interpret the wearer's intention in advance of the actual movement. This is called 'voluntary control system'. HAL has also a 'robotic autonomous control system' that provides human-like movement based on a robotic system which integrally work together with the 'autonomous control system'. HAL can be applied in various fields such as rehabilitation support and physical training support in medical field [43].

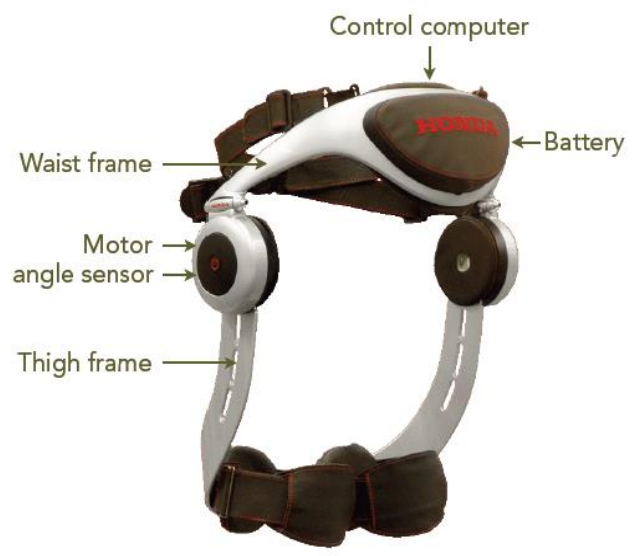

Figure 19. Walking assist device with stride management assist.

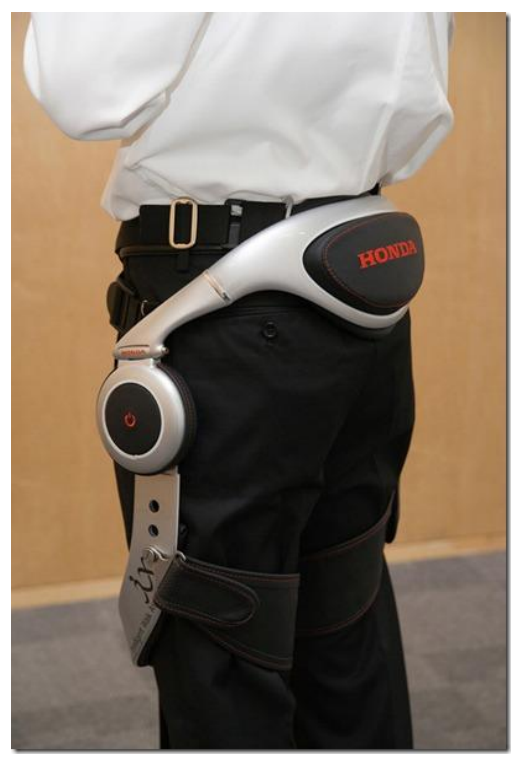

Figure 20. Walking assist device in use.

Walking Assistive Device with Stride Management (Honda Motor) [44] is developed for patients with weakened leg muscles who are still able to walk (Figs 19 and 20).

A motor helps lift each leg at the thigh as it moves forward and backward. This helps lengthen the user's stride, making it easier to cover longer distances at a greater speed. Its lightweight and simple design with a belt worn around the hips and thighs reduces the wearer's load and fits different body shapes. The device weights $2.8 \mathrm{~kg}$ with batteries. It is comprised with 2 brushless DC motors, rechargeable Lithium ion battery, angle sensors, control computer and operates about 2 hours.

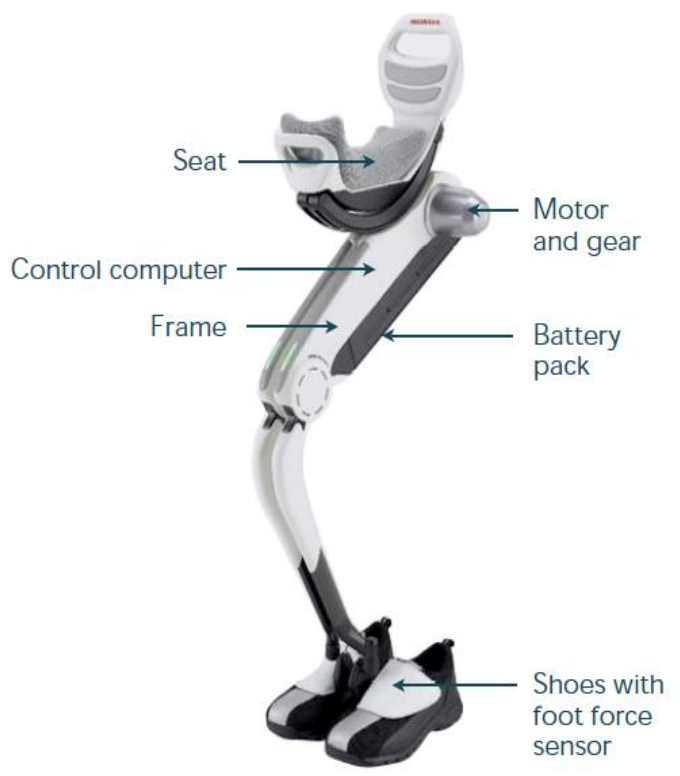

Figure 21. Walking assist device bodyweight support. 
Walking Assist Device with Bodyweight Support Assist (Honda Motor) is shown in Figs. 21 and 22 [44]. Honda's second experimental walking assist device helps support bodyweight to reduce the load on the user's legs while walking. This could lead to reduced fatigue and less physical exertion.

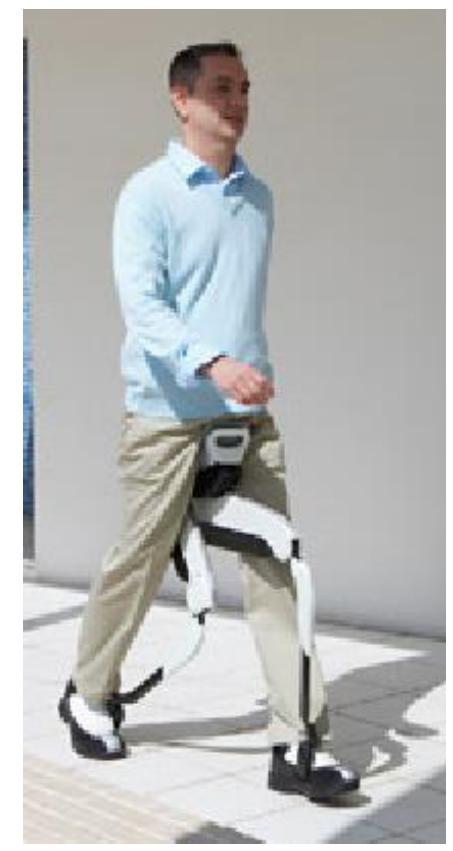

Figure 22. Walking assist device in use.

Honda's device lightens the load on the user's legs and helps maintain a center of gravity via special mechanisms developed by the company. Walking, crouching, climbing stairs all become easier with the help of this device. There are plenty of use cases for this product, not the least of which would be helping people afflicted with mobility issues or leg problems. It can also be used for rehabilitation.

\section{CONCLUSION}

This paper presents an overview of rehabilitation devices of human extremities considering their design concepts and functional particularities. It is obvious that strict space limitations did not permit the inclusion in this review of all the devices worthy of attention. However, we have tried, wherever possible, to describe the most innovative solutions.

Analysis of rehabilitation devices showed that the stationary rehabilitation devices, which are based on the motorized orthosis provide more effective ways to reinstate mobility of the joints. They offer the possibility to adjust the level of gait assistance for each extremity according to the established program. The main advantages of these devices are the possibility of combining mechanical motion generation and controlled electrostimulations. The electric pulses stimulate the muscles, causing them to contract at the appropriate time, which accelerate the rehabilitation period.
However, due to the fast development of high technologies and their applications in robotic rehabilitation, the design of mobile rehabilitation devices is also a promising field. The use of active and passive actuations allows a significant reduction of the size and weight of portative rehabilitation devices. The compact and lightweight characteristics of these advanced rehabilitation devices allow them to be used not only for medical purposes but also as walking assistive devices.

\section{REFERENCES}

[1] http://www.musclepower.com/

[2] http://www.sci-step.com/equipment.htm

[3] http://www.hocoma.com/

[4] http://www.lokohelp.net/fr/produits/lokohelp-pedago/

[5] Freivogel S., Mehrholz J., Husak-Sotomayor T. and Schmalohr D., 2008. "Gait training with the newly developed "LokoHelp"-system is feasible for nonambulatory patients after stroke, spinal cord and brain injury. A feasibility study," Brain Injury, vol. 22, no. 7-8, pp. 625-632.

[6] Freivogel S., Schmalohr D. and J. Mehrholz, 2009. "Improved walking ability and reduced therapeutic stress with an electromechanical gait device," Journal of Rehabilitation Medicine, vol. 41, no. 9, pp. 734-739.

[7] Métrailler P., Brodard R., Stauffer Y., Clavel R. and Frischknecht R., 2007. "Cyberthosis: Rehabilitation Robotics With Controlled Electrical Muscle Stimulation," Rehabilitation Robotics, pp. 303-318.

[8] Métrailler P., 2005. "Système robotique pour la mobilization des membres infèrieurs d'une personne paraplégique," Ecole Polytechnique Fédérale de Lausanne.

[9] Bouri M., Le Gall B. and Clavel R., 2009. “A new concept of parallel robot for rehabilitation and fitness: the Lambda," in Proceedings of the IEEE International Conference on Robotics and Biomimetics, (ROBIO '09), Guilin, China, December, pp. 2503-2508.

[10] Homma K., Fukuda O., Sugawara J., Nagata Y. and Usuba M., 2003. "A wire-driven leg rehabilitation system: development of a 4-dof experimental system," in Proceedings of the International Conference on Advanced Intelligent Mechatronics, (IEEE/ASME '03), vol. 2, Piscataway, NJ, USA, July, pp. 908-913.

[11] Schmidt H., 2004. "HapticWalker - A novel haptic device for walking simulation," in Proceedings of EuroHaptics, June 5-7, pp. 60-67.

[12] Veneman J. F., Kruidhof R., Hekman E. E. G., Ekkelenkamp R., Edwin H. F. Van Asseldonk, and Herman van der Kooij., 2007. "Design and Evaluation of the LOPES Exoskeleton Robot for Interactive Gait Rehabilitation," IEEE Transactions on Neural Systems and Rehabilitation Engineering, September, vol. 15, no. 3, pp. 379-386.

[13] http://www.reha-stim.de 
[14] Galvez J. A. and Reinkensmeyer D. J., 2005. "Robotics for Gait Training After Spinal Cord Injury," Top Spinal Cord Injury Rehabilitation, vol. 11, no. 2, pp. 18-33.

[15] http://www.deaconess.com/bodyPRT.cfm?ID=2295\&

[16] Reinkensmeyer D., Wynne J. and Harkema S., 2002. "A robotic tool for studying locomotor adaptation and rehabilitation," in Proceedings of the Second Joint Meeting of the IEEE Engineering in Medicine and Biology Society and the Biomedical Engineering Society, October 23-26, pp. 2353-2354.

[17] Aoyagi D., IchinoseW. E., Herkema S. J., Reinkensmeyer D. J., and. Bobrow J. E., 2005. "An Assistive Robotic Device That Can Synchronize To The Pelvic Motion During Human Gait Training," in Proceedings of the IEEE 9th International Conference on Rehabilitation Robotics, Chicago, IL, USA, June 28-July 1, pp. 565-568.

[18] Reinkensmeyer D., Aoyagi D., Emken J., et al., 2006. "Tools for understanding and optimizing robotic gait training," Journal of Rehabilitation Research \& Development, August/September, vol. 43, no. 5, pp. 657670.

[19] Surdilovic D, Bernhardt R, Schmidt T, Zhang J., 2004. "String-man: A novel wire robot for gait rehabilitation," Bien ZZ, Stefanov D, eds. Advances in Rehabilitation Robotics. Lecture Notes in Control and Information Sciences. Springer-Verlag, Berlin. pp. 413-424.

[20] Agrawal S. K., Gardner G. and Pledgie S., 2001. "Design and fabrication of a gravity balanced planar mechanism using auxiliary parallelograms," ASME Transactions. Journal of Mechanical Design, vol. 123, no. 4, pp. 525528.

[21] Cardoso L. F., Tomazio S. and Herder J. L., 2002. "Conceptual design of a passive arm orthosis," in Proccesings of the ASME Design Engineering Technical Conference, MECH-34 285, pp. 1-10.

[22] Rahman T., Sample W., Seliktar R., Alexander M. and Scavina M., 2000. "A body-powered functional upper limb orthosis," Journal of Rehabilitation Research and Development, vol. 37, no. 6, pp. 675-680.

[23] Herder J. L., 2005. "Development of a Statically Balanced Arm Support: ARMON," in Proceedings of the IEEE 9th International Conference on Rehabilitation Robotics, June 28 - July 1, Chicago, IL, USA.

[24] Banala S.K., Agrawal S.K., Fattah A., Krishnamoorthy V., Hsu W. L., Scholz J. and Rudolph K., 2006. "GravityBalancing Leg Orthosis and Its Performance Evaluation," IEEE Transactions on Robotics, vol. 22, no. 6, pp. 12281239.

[25] Agrawal S.K. and Fattah A., 2004. "Theory and Design of an Orthotic Device for Full or Partial Gravity-Balancing of a Human Leg During Motion," IEEE Transactions on Neural systems and Rehabilitation Engineering, vol. 12, no. 2, pp. 157-165.

[26] Banala S., Agrawal S. K., Fattah A., 2004. "A gravity balancing leg orthosis for robotic rehabilitation," in
Processing of the International Conference on Robotics and Automation, pp. 2427-2479.

[27] Agrawal S. K., Fattah A., 2004. "Theory and design of an orthotic device for full or partial gravity-balancing of a human leg during motion," IEEE Transactions on Neural Systems and Rehabilitation Engineering, vol. 12, no. 2, pp. 157-165.

[28] Fattah A., Agrawal S. K., 2005. "On the design of a passive orthosis to gravity balance human legs," ASME Transactions. Journal of Mechanical Design, vol. 127, pp. 802-808.

[29] Agrawal A., Agrawal S., 2005. "Design of gravity balancing leg orthosis using non-zero free length springs," Mechanism and Machine Theory, vol. 40, pp. 693-709.

[30] Arakelian V., Ghazaryan S., 2008. "Improvement of balancing accuracy of robotic systems: application to leg orthosis for rehabilitation Devices," Mechanism and Machine Theory, vol. 43, no. 5, pp. 565-575.

[31] Arakelian V., Ghazaryan S., 2006. "Gravity balancing of the human leg taking into account the spring mass," in Proceedings of the 9th International Conference on Climbing and Walking Robots (CLAWAR), Brussels, Belgium, 12-14 September, pp. 630-635.

[32] Banala S. K., Kim S. H., Agrawal S. K. and Scholz J. P., 2009. "Robot Assisted Gait Training With Active Leg Exoskeleton (ALEX)," IEEE Transactions on Neural Systems and Rehabilitation Engineering, vol. 17, no. 1, February, pp. 2-8.

[33] Onogi K., Kondo I., Saitoh E.,Kato M. and Oyobe T., 2010. "Comparison of the effects of sliding-type and hinge-type joints of knee-ankle-foot orthoses on temporal gait parameters in patients with paraplegia," Japanese Journal of Comprehensive Rehabilitation Science, vol. 1, pp. 1-6.

[34] http://www.scheckandsiress.com/

[35] http://www.litegait.com/

[36] http://www.ropox.com/

[37] http://www.sigmedics.com/

[38] http://www.berkelbike.co.uk/

[39] http://www.yobotics.com

[40] Pratt J. E., Collins S. H., Krupp B. T. and Morse C. J., 2004. "The RoboKnee: An Exoskeleton for Enhancing Strength and Endurance During Walking," Proceeding of the IEEE International Conference on Robotics \& Automation, New Orleans, LA, April, pp. 2430-2435.

[41] Dollar M. A. and Herr, H., 2008. "Design of a QuasiPassive Knee Exoskeleton to Assist Running," Acropolis Convention Center, Nice, France, September 22-26, pp. 747-754.

[42] Sankai Yoshiyuki, "HAL: Hybrid Assistive Limb based on Cybernics," Global COE Cybernics, System and Information Engineering, University of Tsukuba, Japan, pp. 1-10.

[43] http://www.cyberdyne.jp/english/

[44] http://www.walkassist.honda.com/ 